\title{
Haematopoietic cell transplantation as immunotherapy
}

\author{
Frederick R. Appelbaum
}

Clinical Research Division, Fred Hutchinson Cancer Research Center, 1100 Fairview Avenue North, D5-310, PO Box 19024, Seattle, Washington 98109-1024, USA

The graft-versus-tumour effect seen after allogeneic (genetically different) haematopoietic cell transplantation for human malignancies represents the clearest example of the power of the human immune system to eradicate cancer. Recent advances in our understanding of the immunobiology of stem-cell engraftment, tolerance and tumour eradication are allowing clinicians to better harness this powerful effect.

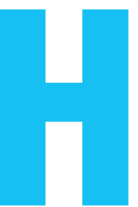

igh-dose

systemic

chemotherapy or chemoradiotherapy followed by allogeneic haematopoietic cell transplantation (HCT) can be an effective therapy for many patients with otherwise fatal haematological malignancies. Cure rates vary, but range from as high as $80 \%$ for patients with chronic myeloid leukaemia (CML) treated during chronic phase to only $15-20 \%$ for patients with acute leukaemia refractory to conventional chemotherapy. Despite its limitations and toxicity, allogeneic transplantation is sufficiently effective that it was used to treat approximately 18,000 patients worldwide last year alone.

The initial rationale for HCT came from laboratory and clinical observations that most haematological malignancies exhibit a steep dose-response reaction to alkylating agents and radiation therapy. Because marrow toxicity is dose limiting for many of these agents, by transplanting pluripotent haematopoietic stem cells contained in bone marrow or peripheral blood, it became possible to administer far higher doses of therapy than was otherwise possible. It has since become apparent that immunocompetent cells transplanted with the stem cells, or arising from them, exert a potent graft-versus-tumour effect independent of the effects of the high-dose therapy.

Barnes et al. first suggested the existence of a graftversus-tumour (GVT) effect in 1956 when they noted eradication of leukaemia in irradiated mice receiving allogeneic marrow transplants, but not syngeneic transplants from identical twins ${ }^{1}$. The initial evidence for such an effect in humans came from studies reporting that relapse rates following allogeneic transplantation were markedly less in patients who developed graft-versus-host disease (GVHD) compare with those who did not ${ }^{2,3}$. Subsequent studies revealed that relapse rates are least in patients who develop both acute and chronic GVHD, higher in those who develop no clinically evident GVHD, and higher still if T cells are depleted from the marrow graft or in recipients of twin transplants (see Fig. 1) ${ }^{4}$. Further verification of the GVT effect came from efforts to treat patients for post-transplant leukaemic recurrence by infusing donor lymphocytes in hopes of inducing GVHD and an associated anti-tumour effect. Somewhat surprisingly, sustained complete responses were seen in most patients with CML, and in many patients with other haematological malignancies ${ }^{5}$. With increased recognition of the strength of the GVT effect and the recent development of methods to better exploit it, clinical research is beginning to focus on allogeneic HCT more as an immunotherapeutic approach, rather than solely a vehicle to deliver high-dose therapy.

\section{Histocompatibility and transplantation}

Transplantation of allogeneic haematopoietic cells is accompanied by reciprocal immunological reactions of the graft against its new host and the host against the graft. The human leukocyte antigen (HLA) system, which is the human analogue of a multigene system known as the major histocompatibility complex (MHC), is crucial in the development of these reactions ${ }^{6}$. Located on chromosome 6 , it spans more than 4 megabases and includes more than 200 genes. For allogeneic HCT, the most influential genes are $H L A-A, H L A-B$ and $H L A-C$, collectively referred to as class I genes, and $D R B 1, D Q B 1$ and $D P B 1$, collectively referred to as class II genes. The class I genes are expressed on virtually all nucleated cells, whereas expression of class II genes is restricted largely to cells of the immune system. These genes are highly polymorphic; more than 125 HLA-A, 260 HLA-B, 75 HLA-C, 225 HLA-DRB1 and 40 HLA-DQB1 alleles have been described ${ }^{7}$.

HLA molecules are fundamental in T-cell activation, as they bind peptides and present them to T cells. HLA class I molecules preferentially present peptides to $\mathrm{CD}^{+} \mathrm{T}$ cells, whereas $\mathrm{CD} 4^{+} \mathrm{T}$ cells preferentially recognize peptides presented by HLA class II molecules ${ }^{8,9}$. The HLA molecules themselves are termed major histocompatibility antigens and $\mathrm{T}$ cells confronting non-identical HLA molecules react vigorously. The peptides presented by HLA molecules may come from external sources (for example, viruses), but mostly they derive from endogenous proteins. During normal maturation of the immune system, tolerance develops to these 'self' proteins. However, in the context of organ transplantation, polymorphisms in these endogenous proteins serve as sources of minor histocompatibility antigens and form the basis of immunological non-identity between HLA-matched individuals ${ }^{10}$.

The immunological non-identity between donor and recipient has three main consequences for the use of allogeneic HCT as immunotherapy. First, after transplantation, the host may mount an immunological attack against the graft, leading to graft rejection. Because engraftment is required to exert a GVT response, methods to assure sustained engraftment are necessary. Second, immunocompetent cells in the graft can react against antigens of normal host tissues, which can result in life-threatening or even fatal GVHD. For the safe application of HCT, this reaction must 
be controlled. Third, the GVT effect has been closely intertwined with the development of GVHD. If GVHD is to be controlled and the GVT effect strengthened, strategies to separate the two are required.

\section{Engraftment and non-myeloablative transplants}

Studies performed more than three decades ago using outbred species matched for major histocompatibility antigens found that very high dose chemotherapy or systemic radiotherapy administered to the recipient pretransplant was necessary to eradicate host $\mathrm{T}$ cells sufficiently to prevent graft rejection ${ }^{11}$. Thus, until recently, most transplant 'preparative' regimens included marrow-ablative doses of therapy, not only for their anti-tumour effect, but also to ensure sustained engraftment. The intensity of these regimens limited the application of transplantation to younger, relatively healthy patients and made it difficult to distinguish the anti-tumour effects of the graft from those of the intensive preparative regimen.

With the development of more specifically immunosuppressive chemotherapeutic agents, such as fludarabine, and increased appreciation of the GVT effect, investigators have begun exploring less intensive 'non-myeloablative' preparative regimens, for example, fludarabine with moderately high dose melphalan or busulphan. Initial studies report sustained engraftment in recipients of grafts from HLA-matched siblings, diminished toxicity compared to conventional approaches, and long-term disease-free survival in a proportion of patients ${ }^{12-14}$.

Although these studies focused largely on pretransplant cytotoxic therapy to enable engraftment, post-transplant treatment of the graft recipient with potent immunosuppression contributes significantly to preventing graft rejection. One set of experiments is shown in Table 1. Using the model of DLA (the canine equivalent of HLA)identical littermates, Storb et al. showed that if no post-transplant immunosuppression is given, dogs require 920 cGy total body irradiation (TBI) to engraft. But if two potent immunosuppressive agents are given post-transplant, the dose of TBI required to achieve engraftment falls to $200 \mathrm{cGy}$, a dose far below that which causes bone marrow aplasia $^{15}$. These observations prompted studies of conditioning regimens of very limited intensity in humans, to determine whether, as in animals, engraftment could be achieved with such low-dose therapy, and if so, what extent of tumour response would follow.

Initial clinical trials by our group involved patients who were not candidates for conventional transplants because of age or other medical problems, but who had haematological malignancies that were otherwise appropriate for transplantation. The initial treatment plan followed the animal model, and involved pretransplant treatment of patients with $200 \mathrm{cGy}$ TBI followed post-transplant by the administration of mycophenolate mofetil and cyclosporine. Because occasional cases of graft rejection were seen in the first cohort of patients, low-dose fludarabine was added to the pretransplant regimen. Once fludarabine was added, graft rejection ceased to be a problem. Results in the first 109 patients have so far been reported ${ }^{16,17}$. These patients (median age 55) had a variety of otherwise incurable haematological malignancies, but tolerated the transplant procedure well. Fiftyseven percent were treated entirely as outpatients, with the remaining requiring hospitalizations averaging approximately one week, as compared with an average hospitalization of over one month with conventional transplantation. The treatment-related death rate over the first 3 months was $4.5 \%$, substantially less than the $15-20 \%$ rates seen in younger patients treated with conventional myeloablative transplant regimens. Sixty-six percent of patients who had measurable tumour before transplant achieved a complete response with this treatment. Responses were seen in virtually all categories of haematological malignancy, but were most frequent and enduring in patients with less rapidly proliferative diseases such as CML, chronic lymphocytic leukaemia and nodular lymphoma, perhaps reflecting the kinetics of the GVT response.

Non-haematological malignancies have also responded to similar low-intensity transplant approaches. Childs et al. reported that 10 of

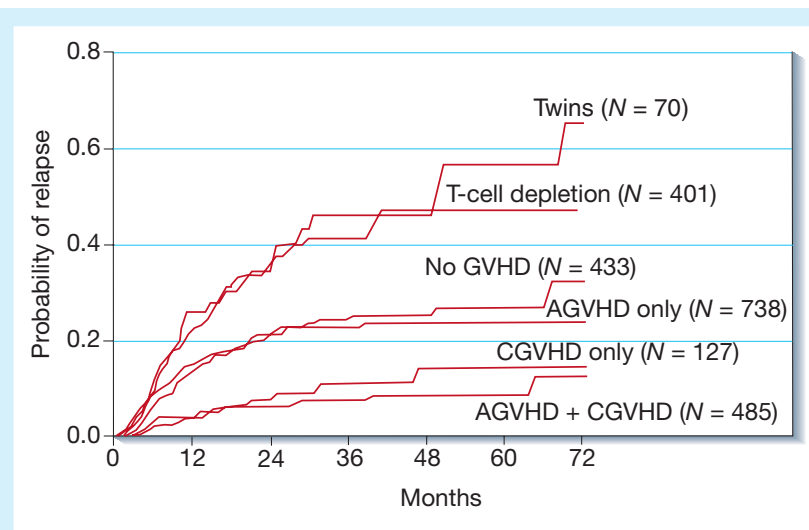

Figure 1 Relapse rates following allogeneic and syngeneic marrow transplantation. Relapse rates are least in patients who develop both acute and chronic graft-versushost disease (AGVHD + CGVHD), higher in those who develop no clinically evident GVHD, and higher still if T cells are depleted from the marrow graft or in recipients of twin transplants ${ }^{4}$.

19 patients $(53 \%)$ with metastatic renal-cell carcinoma exhibited disease regression, including three complete responses that have endured for periods beyond 2 years ${ }^{18}$.

These studies show that engraftment of allogeneic haematopoietic cells can be achieved with very low dose therapy and can result in pronounced anti-tumour effects. However, the procedure is also accompanied by significant GVHD in approximately $50 \%$ of individuals, and anti-tumour responses are frequently less than complete. Thus, methods both to prevent GVHD and to augment the GVT effect are required.

\section{Induction of immunological tolerance}

Because of the importance of HLA compatibility in the outcome of transplantation, most allogeneic transplants have been between HLA-matched individuals. Before 1980, this exclusively meant HLAmatched siblings, but only one in three patients have such donors available. Since that time, approximately 6.5 million normal individuals have been HLA-typed as potential unrelated volunteer marrow donors. This allows for the identification of HLA-matched unrelated donors for over $50 \%$ of patients lacking matched siblings.

GVHD results from T cells transplanted with the graft or developing from it reacting with major or minor histocompatibility antigens of the genetically different host. The development of clinically significant GVHD, although associated with a reduced risk of leukaemic relapse, leads to poorer overall survival owing to the direct effects of the disease and the consequences of the immunosuppression used to treat $\mathrm{it}^{19}$. Conventional methods to prevent GVHD have relied on a combination of the antimetabolite methotrexate given early after transplant to kill donor $\mathrm{T}$ cells responding vigorously to host antigens, along with cyclosporine, which blocks a calcium-dependent signal-transduction pathway distal to engagement of the T-cell receptor. Despite such prophylaxis, significant GVHD develops in $40 \%$ of patients transplanted from matched siblings and $70 \%$ of recipients of matched unrelated transplant $\mathrm{s}^{20}$.

The higher incidence of GVHD in recipients of unrelated transplants has variously been ascribed to unrecognized incompatibilities in major histocompatibility antigens or greater heterogeneity in minor histocompatibility antigens. Before 1998, HLA typing was largely dependent on serologic methods, which do not identify all differences. More recently, studies have been conducted in which HLA-A, -B, -C, DRB1 and DQB1 have been analysed at the allele level using automated direct sequencing. These studies detected allele-level mismatches in over $30 \%$ of serologically matched donor-recipient pairs ${ }^{21}$. Allele-level mismatching at class I antigens is associated with an increased incidence of graft rejection, but has no 
impact on GVHD, whereas mismatching at class II is associated with increased GVHD without effect on graft rejection ${ }^{21,22}$. Overall survival is markedly less in patients with multiple class I allele-level mismatches and in those with both class I and class II mismatches. Although allele-level matching should improve survival, completely matched donors will not be available for all patients, and so efforts are being made to identify those mismatches that are permissive and not associated with increased GVHD or graft rejection.

A substantial amount of work has focused on T-cell depletion (TCD) of the donor stem-cell graft as a method of preventing $\mathrm{GVHD}^{23}$. A number of techniques exist for removing T cells, most of which use antibodies (complement mediated lysis, immunotoxins and immunomagnetic beads) or physical methods (soybean lectin agglutination, counter-flow elutriation and albumin-gradient fractionation). Clinical studies using these approaches have shown unambiguously that TCD markedly reduces the incidence and severity of GVHD. However, TCD is associated with an increased rate of severe and often fatal infections, a higher incidence of graft rejection, and an increased risk ofleukaemia recurrence.

The increase in infectious complications is explained by studies showing that very few $\mathrm{CD} 4^{+}$and $\mathrm{CD} 8^{+} \mathrm{T}$ cells develop from the transplanted stem cell over the first three months after transplant and, therefore, T-cell immunity during this period is dependent on $\mathrm{T}$ cells transplanted with the stem cells ${ }^{24}$. The increased incidence of graft failure with TCD probably reflects the loss of the contribution that donor $\mathrm{T}$ cells normally make in eradicating residual host immune cells surviving the transplant preparative regimen. The increased leukaemia relapse rate seen after T-cell depletion (Fig. 1) highlights the importance of the T-cell response in eradicating malignancy. The impact of TCD on graft rejection can be reduced by further intensifying the preparative regimen with additional chemotherapy and antithymocyte globulin ${ }^{25}$. To lessen the impact of TCD on infections and leukaemia relapse, partial TCD, delayed re-infusion of donor lymphocytes, and post-transplant administration of low-dose interleukin (IL)-2 are all being studied ${ }^{26-28}$.

As an alternative to TCD, techniques capable of inducing antigenspecific tolerance shortly after allogeneic HCT are conceptually

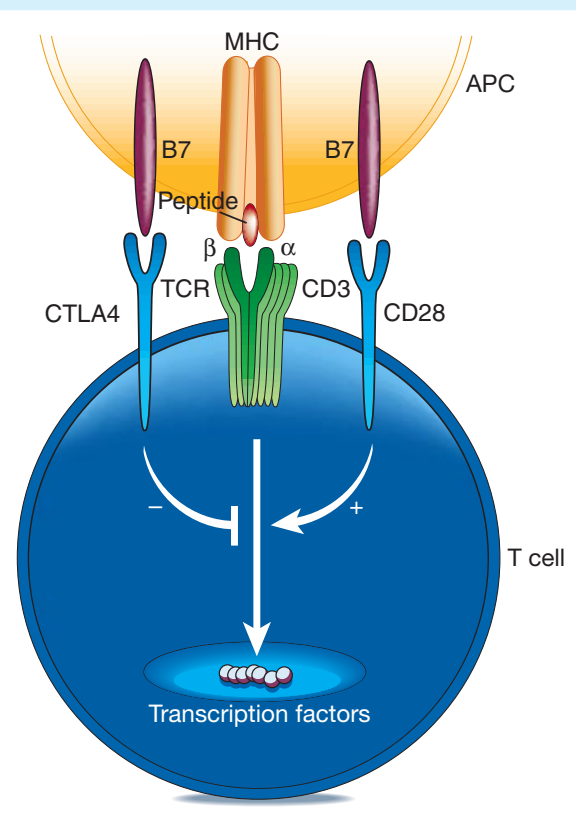

Figure $2 \mathrm{~T}$-cell regulation by CD28 and CTLA4. T-cell activation requires stimulation of the T-cell receptor with its appropriate antigen presented by MHC on the antigenpresenting cell. The degree of T-cell response is regulated by secondary interactions of $\mathrm{B} 7$ molecules on the antigen-presenting cell with $\mathrm{CD} 28$, which leads to activation, or with CTLA4, which results in inhibition of response. appealing in that they would prevent GVHD without resulting in profound post-grafting immunosuppression. One approach to the development of antigen-specific tolerance builds on the observation in murine models that exposure of antigen-activated $\mathrm{T}$ cells to antibodies against the invariant CD3 domain of the T-cell receptor can induce apoptosis specifically in activated cells, thereby preventing $\mathrm{GVHD}^{29}$. Accordingly, a humanized non-FcR-binding anti-CD3 antibody, Hu291, has been developed and is now in clinical trials with promising early results.

A second approach for the development of antigen-specific tolerance is based on the 'two-signal model' of T-cell activation. T-cell activation requires not only stimulation of the $\mathrm{T}$-cell receptor with its appropriate antigen in the context of $\mathrm{MHC}$, but also a second 'co-stimulatory' signal provided by CD28 (Fig. 2). Experiments showed that stimulation of $\mathrm{T}$ cells with antigen plus an activating CD28 antibody in vitro induced IL-2 gene expression, whereas a blocking anti-CD28 antibody caused inactivation of the IL-2 gene ${ }^{30}$. And transplantation using CD28-knockout mice as donors resulted in partial protection of recipients from lethal $\mathrm{GVHD}^{31}$. CD28 binds to two ligands, B7-1 (CD80) and B7-2 (CD86) on antigen-presenting cells (APCs $)^{32}$. These ligands bind an additional T-cell antigen, CTLA4, which is expressed only after T-cell activation. A soluble CTLA4-immunogloblin fusion protein (CTLA4-Ig) has been produced as a competitive inhibitor blocking CD28-B7 interactions. CTLA4-Ig blocks rejection of human pancreatic islet cells in mice and induces long-term, donor-specific tolerance ${ }^{33}$. A possible shortcoming of CTLA4-Ig is that it may also block interactions of B7 and CTLA4 itself, which serves as a negative regulator of T-cell activation $^{34}$. Thus, an alternative approach is to block CD28-B7 interactions directly using a CD28-specific antibody. In murine models, one such antibody has been effective in preventing GVHD, but this approach has yet to be tested in humans $s^{35,36}$.

Other studies have focused on the role of inflammatory cytokines and host APCs in the pathogenesis of GVHD. The intense preparative regimens administered before transplantation and subsequent infections induce secretion of tumour-necrosis factor (TNF)- $\alpha$, IL- 1 and other pro-inflammatory cytokines from APCs and other host tissues, which amplify subsequent alloimmune reactions and lead to greater $\mathrm{GVHD}^{37}$. In murine models, this reaction can be substantially blocked by using specific cytokine antagonists such as IL-1-receptor antagonist or antibodies to TNF, which has led to similar trials in humans. Furthermore, although donor APCs can cross-present host antigens, they seem to do so less effectively than host APCs. Thus, pretransplant elimination of host APCs capable of presenting host antigens via the endogenous pathway can reduce GVHD in murine models ${ }^{38}$. Unfortunately, no practical method exists to accomplish this in humans.

\section{Segregating anti-tumour from anti-host reactions}

The potency of the GVT effect, coupled with the direct demonstration of complete tumour responses following infusion of donor $\mathrm{T}$ cells for post-transplant relapse, has fuelled interest in the development of T-cell therapy to treat haematological malignancies. Clinical trials, in which T cells specific for cytomegalovirus (CMV), human immunodeficiency virus, Epstein-Barr virus and melanoma antigens were adoptively transferred, have established the safety of this approach and demonstrated that transferred T cells can persist in vivo, migrate to sites of antigen, and exert effector function. For example, using CMV immunity as a model, Riddell et al. isolated $\mathrm{CMV}$-specific $\mathrm{CD}^{+} \mathrm{T}$ cells from marrow donors pre-transplant, expanded the cells in vitro, and administered the cells to marrow transplant recipients, which led to reconstitution of potentially protective $\mathrm{T}$-cell immunity to $\mathrm{CMV}^{39,40}$. Thus, many of the principles required for successful adoptive T-cell therapy have been established. The main challenge now is the identification of antigens that can be used to effectively separate anti-tumour from anti-host reactions. 
Two general categories of antigens capable of serving as targets for T-cell therapy are currently under study — polymorphic minor histocompatibility antigens, and antigens associated with the malignant phenotype. As noted in Fig. 1, the GVT effect can occur in the absence of GVHD, indicating that a subset of minor histocompatibility antigens (mHAgs) expressed by leukaemia cells and recognized by donor $\mathrm{T}$ cells are not expressed by the non-haematopoietic tissues that are targets of GVHD. Thus, one general strategy to segregate GVT from GVHD is to identify polymorphic minor histocompatibility antigens that are differentially expressed by haematopoietic and nonhaematopoietic tissue. Such antigens should be able to serve as targets for post-transplant donor-derived T-cell therapy aimed at ablating all normal and malignant lymphohaematopoietic cells of the host.

In an effort to identify relevant minor histocompatibility antigens, Goulmy and Engelhard used a biochemical approach involving the elution of peptides from class I MHC molecules, separation of peptide fractions by high-performance liquid chromatography, identification of fractions that reconstitute T-cell recognition when pulsed onto target cells, and sequencing of the recognized peptides by mass spectrometry ${ }^{41,42}$. Five human mHAgs have been identified by this approach, three of which are broadly expressed and thus likely to serve as targets both for GVHD and for GVT, and two that seem to be expressed selectively by haematopoietic cells and thus could serve as targets for a GVT-specific response $\mathrm{s}^{43,44}$. Riddell and Warren have used cell-culture techniques to isolate $\mathrm{CD}^{+}{ }^{+} \mathrm{mHAg}$-specific T-cell clones from allogeneic HCT recipients, and have characterized 38 previously undescribed mHAgs ${ }^{45,46}$. A substantial number of these antigens seem to be restricted in their expression to haematopoietic tissues, as T-cell clones lyse recipient cells of haematopoietic origin but not recipient fibroblasts.

Acute myeloid leukaemia (AML) consists of a hierarchy of cells with different capacities for self-renewal. Transplantation of human AML into NOD/SCID mice has identified a potential leukaemia stem cell, termed the SCID leukaemia-initiating cell ${ }^{47}$. Whether any specific mHAg identified by existing screening will be expressed by the patient's leukaemic stem cell is uncertain, but can be inferred by measuring the ability of $\mathrm{T}$ cells specific for such an antigen to eliminate human AML in the NOD/SCID mouse. When this was tested, outgrowth of AML was specifically prevented ${ }^{48}$.

Clinical trials testing the safety and efficacy of T-cell clones specific for mHAgs have been initiated for patients with leukaemia in relapse after allogeneic transplantation. Because it is not possible to screen all normal tissues for expression of the targeted antigen, T-cell clones for initial infusion have been transfected with a herpes simplex thymidine-kinase suicide gene. Early results suggest that administration of such cells can be associated with achievement of complete remission without necessarily augmenting GVHD (ref. 44, and S. R. Riddell, personal communication).

Although polymorphic minor histocompatibility antigens are rational targets for separating GVT from GVHD, they will always require the context of allogeneic HCT for their use. An alternative approach is to identify antigens associated with the malignant phenotype. Such antigens could serve as targets for T-cell therapy, not only following allogeneic transplantation, but also in other settings. Candidate antigens can be classified as: (1) mutational, such as $\mathrm{Bcr} / \mathrm{Abl}$, which should be tumour specific; (2) viral, such as human papillomavirus in cervical cancer, which should also be tumour specific; (3) tissue specific, such as prostate-specific antigen in prostate cancer, where destruction of normal tissue is permissible; (4) germ-cell antigens, such as the melanoma-associated antigen family, normally expressed in adults only in the testes; and (5) overexpressed self-proteins, which may be recognized based on increased levels of presentation. The immunogenicity of such antigens is influenced by many factors, including level of protein expression, the peptide sequences resulting from intracellular processing, and the ability of these peptides to be presented by class I molecules. Host responses to such antigens may range from non-responsiveness

\begin{tabular}{llc}
\hline Table 1 Marrow grafts from DLA-identical canine littermates after TBI \\
\hline $\begin{array}{l}\text { TBI dose } \\
\text { (cGy) }\end{array}$ & $\begin{array}{l}\text { Postgrafting } \\
\text { immunosuppression }\end{array}$ & No. engrafted/No. studied \\
\hdashline 920 & - & $20 / 21$ \\
\hline 450 & - & $16 / 39$ \\
\hline 450 & CSP & $7 / 7$ \\
\hline 200 & CSP & $0 / 4$ \\
\hline 100 & MMF/CSP & $11 / 12$ \\
\hline
\end{tabular}

Abbreviations: TBI, total body irradiation; CSP, cyclosporine; MMF, mycophenolate mofetil.

resulting from prior tolerance, to production of T cells of sufficient avidity to selectively destroy targeted cells.

Mutational antigens considered as targets for T-cell therapy include $\mathrm{Bcr} / \mathrm{Abl}$ in CML and PML/RAR $\alpha$ in acute promyelocytic leukaemia. However, reproducible generation of $\mathrm{CD}^{+} \mathrm{T}$ cells that recognize leukaemia progenitor cells bearing these antigens has not yet been possible ${ }^{49,50}$. Normal proteins overexpressed in leukaemia progenitors may provide alternative targets. Ideally, such antigens should be expressed at substantially higher levels in leukaemia than in normal cells, expressed by all leukaemia cells and be efficiently processed and presented by diverse HLA class I molecules. Two proteins that fit this description, proteinase 3 (PR3) and Wilms' tumour-suppressor (WT1), have already been shown to elicit $\mathrm{CD}^{+}$responses.

PR3 is a neutral serine proteinase with expression largely restricted to the promyelocytic stage of myeloid differentiation ${ }^{51,52}$. Although PR3 is not detected in normal haematopoietic stem cells, it is expressed in freshly isolated leukaemia progenitors, particularly from CML patients. CD ${ }^{+} \mathrm{T}$ cells specific for PR3 have been generated by stimulation of cells with a peptide predicted to bind to the A2.1 class I allele $\mathrm{e}^{53,54}$. Such cells selectively lyse leukaemic blasts but not normal bone marrow cells. $\mathrm{CD}^{+}$cytotoxic T lymphocytes (CTLs) specific for this epitope were not detected in the peripheral blood of normal individuals or in untreated CML patients, but they could be found in the blood of CML patients who had been treated successfully with allogeneic transplantation or with $\alpha$-interferon, suggesting a role for these cells in the anti-tumour response ${ }^{55}$.

WT1, a zinc-finger transcription factor, was initially described as a tumour-suppressor gene in childhood Wilms' tumour. WT1 is abundantly overexpressed in most human leukaemia cells, including AML, CML and acute lymphocytic leukaemia, with higher levels associated with a worse prognosis ${ }^{56,57}$. Leukaemia cells express from 10 - to $>100$-fold more WT1 protein than normal CD $34^{+}$cells. Studies indicate that $\mathrm{T}$ cells can distinguish this difference in protein expression, as $\mathrm{CD}^{+}$CTLs generated against WT1 lyse leukaemic $\mathrm{CD} 34^{+}$but not normal CD $34^{+}$cells, and inhibit growth of leukaemic but not normal myeloid colonies ${ }^{58}$. Thus, like PR3, WT1 might serve as a useful target for adoptive T-cell therapy. Development of gene microarrays, which enable the expression of thousands of genes simultaneously, should facilitate the identification of additional proteins that are overexpressed in leukaemia progenitors.

Success in the application of T-cell therapy will have important implications for alternative strategies, particularly vaccine development. Murine studies have, in fact, shown enhanced immune responses to a vaccine based on granulocyte-macrophage colony-stimulating factor administered in the early post-transplant period, compared to the non-transplant setting ${ }^{59}$. Direct T-cell transfer should greatly aid vaccine development by defining the therapeutic efficacy of targeting any specific antigen and any possible toxicity to normal tissues.

\section{Summary}

The ability to achieve complete haematopoietic engraftment without intensive therapy will have a profound effect on the practise of allogeneic HCT. Rather than treating patients with high-dose preparative regimens to both eradicate the malignancy and prevent graft rejection, efforts to capture the benefits of high-dose therapy can 
focus on developing treatments specifically targeted to tumour eradication and combining these with specific immunosuppression to ensure engraftment. For example, studies are now underway combining high-dose monoclonal antibody-targeted radiotherapy with non-myeloablative transplant regimens ${ }^{60}$. Methods to induce antigenspecific tolerance following transplantation promise to reduce GVHD without producing severe prolonged immunodeficiency. Finally, and perhaps most important, strategies now exist to segregate GVT from GVHD. The identification of a modest number of polymorphic minor histocompatibility antigens with expression limited to the lymphohaematopoietic system should allow augmentation of the GVT response in most patients transplanted for haematological malignancies, either by adoptive transfer of T cells, or perhaps, vaccination of the donor before transplant. If the adoptive transfer of $\mathrm{T}$ cells specific for overexpressed tumour antigens proves safe and effective, as preclinical experiments predict, this will encourage study of adoptive T-cell transfer in both transplant and non-transplant settings, and pave the way for vaccine trials. Furthermore, with the rapid application of gene microarray analyses, additional new candidate antigens will probably become apparent for multiple tumour types.

1. Barnes, D. W. H., Corp, M. J., Loutit, J. F. \& Neal, F. E. Treatment of murine leukaemia with x-rays and homologous bone marrow. Preliminary communication. Br. Med. J. 2, 626-627 (1956).

2. Weiden, P. L. et al. Antileukemic effect of graft-versus-host disease in human recipients of allogeneicmarrow grafts. N. Engl. J. Med. 300, 1068-1073 (1979).

3. Weiden, P. L., Sullivan, K. M., Flournoy, N., Storb, R., Thomas, E. D. \& the Seattle Marrow Transplant Team. Antileukemic effect of chronic graft-versus-host disease. Contribution to improved survival after allogeneic marrow transplantation. N. Engl. J. Med. 304, 1529-1533 (1981).

4. Horowitz, M. M. et al. Graft-versus-leukemia reactions after bone marrow transplantation. Blood 75, 555-562 (1990)

5. Kolb, H. J. et al. Graft-versus-leukemia effect of donor lymphocyte transfusions in marrow grafted patients. European Group for Blood and Marrow Transplantation Working Party Chronic Leukemia. Blood 86, 2041-2050 (1995).

6. Klein, J. \& Sato, A. The HLA system. N. Engl. J. Med. 343, 702-709, 782-786 (2000).

7. Bodmer, J. G. et al. Nomenclature for factors of the HLA system, 1998. Tissue Antigens 53, 407-446 (1999).

8. Doyle, C. \& Strominger, J. L. Interaction between CD4 and class II MHC molecules mediates cell adhesion. Nature 330, 256-259 (1987).

9. Norment, A. M., Salter, R. D., Parham, P., Engelhard, V. H. \& Littman, D. R. Cell-cell adhesion mediated by CD8 and MHC class I molecules. Nature 336, 79-81 (1988).

10. Parham, P. Deconstructing the MHC. Nature 360, 300-301 (1992).

11. Storb, R. et al. Marrow grafts between DL-A-matched canine littermates. Transplantation 15 , 92-100 (1973).

12. Khouri, I. F. et al. Transplant-lite: induction of graft-versus-malignancy using fludarabine-based nonablative chemotherapy and allogeneic blood progenitor-cell transplantation as treatment for lymphoid malignancies. J. Clin. Oncol. 16, 2817-2824 (1998).

13. Slavin, S., Nagler, A., Aker, M., Shapira, M. \& Or, R. in New Frontiers in Cancer Therapy: Nonmyeloablative Stem Cell Transplantation (NST) (eds Giralt, S. \& Slavin, S.) 83-91 (Darwin Scientific Publishing, Oxford, 2000).

14. Giralt, S. et al. Melphalan and purine analog-containing preparative regimens: reduced-intensity conditioning for patients with hematologic malignancies undergoing allogeneic progenitor cell transplantation. Blood 97, 631-637 (2001).

15. Storb, R., Yu, C. \& McSweeney, P. in Hematopoietic Cell Transplantation, 2nd edn (eds Thomas, E. D., Blume, K. G. \& Forman, S. J.) 287-295 (Blackwell Science, Boston, 1999).

16. McSweeney, P. A. et al. Hematopoietic cell transplantation in older patients with hematologic malignancies: replacing high-dose cytotoxic therapy with graft-versus-tumor effects. Blood (in the press)

17. Sandmaier, B. M. et al. Nonmyeloablative conditioning for HLA-identical related allografts for hematologic malignancies. Blood 96, 479a (abstract 2062) (2000).

18. Childs, R. et al. Regression of metastatic renal-cell carcinoma after nonmyeloablative allogeneic peripheral-blood stem-cell transplantation. N. Engl. J. Med. 343, 750-758 (2000).

19. Nash, R. A. et al. Acute graft-versus-host disease: analysis of risk factors after allogeneic marrow transplantation and prophylaxis with cyclosporine and methotrexate. Blood 80, 1838-1845 (1992).

20. Sullivan, K. M. in Bone Marrow Transplantation (eds Forman, S. J., Blume, K. G. \& Thomas, E. D.) 339-362 (Blackwell Scientific Publications, Boston, 1994).

21. Petersdorf, E. W. et al. Optimizing outcome after unrelated marrow transplantation by comprehensive matching of HLA class I and II alleles in the donor and recipient. Blood 92, 3515-3520 (1998).

22. Petersdorf, E. W. et al. Association of HLA-C disparity with graft failure after marrow transplantation from unrelated donors. Blood 89, 1818-1823 (1997).

23. Kernan, N. A. in Bone Marrow Transplantation (eds Forman, S. J., Blume, K. G. \& Thomas, E. D.) 124-135 (Blackwell Scientific Publications, Boston, 1994).

24. Storek, J. \& Storb, R. T-cell reconstitution after stem-cell transplantation-by which organ? Lancet 355, 1843-1844 (2000).

25. Papadopoulos, E. B. et al. T-cell-depleted allogeneic bone marrow transplantation as postremission therapy for acute myelogenous leukemia: freedom from relapse in the absence of graft-versus-host disease. Blood 91, 1083-1090 (1998).
26. Soiffer, R. J., Murray, C., Gonin, R. \& Ritz, J. Effect of low-dose interleukin-2 on disease relapse after T-cell depleted allogeneic bone marrow transplantation. Blood 84, 964-971 (1994).

27. Champlin, R. et al. Selective depletion of CD8+ T lymphocytes for prevention of graft-versus-host disease after allogeneic bone marrow transplantation. Blood 76, 418-423 (1990).

28. Dazzi, F. et al. Comparison of single-dose and escalating-dose regimens of donor lymphocyte infusion for relapse after allografting for chronic myeloid leukemia. Blood 95, 67-71 (2000).

29. Anasetti, C., Bidwell, B. J., Martin, P. J. \& Yu, X. Z. Anti-CD3 F(AB')2 prevents graft-versus-host disease by selectively depleting donor T cells activated by recipient alloantigens. Blood $96,582 \mathrm{a}$ (abstract 2499) (2000).

30. Harding, F. A., McArthur, J. G., Gross, J. A., Raulet, D. H. \& Allison, J. P. CD28-mediated signalling costimulates murine T cells and prevents induction of anergy in T-cell clones. Nature 356, 607-609 (1992).

31. Yu, X.-Z., Martin, P. J. \& Anasetti, C. Role of CD28 in acute graft-versus-host disease. Blood 92, 2963-2970 (1998).

32. Lenschow, D. J., Walunas, T. L. \& Bluestone, J. A. CD28/B7 system of T cell costimulation. Annu. Rev. Immunol. 14, 233-258 (1996).

33. Lenschow, D. J. et al. Long-term survival of xenogeneic pancreatic islet grafts induced by CTLA4lg. Science 257, 789-792 (1992).

34. Perez, V. L. et al. Induction of peripheral T cell tolerance in vivo requires CTLA-4 engagement. Immunity 6, 411-417 (1997).

35. Yu, X. Z., Bidwell, S. J., Martin, P. J. \& Anasetti, C. CD28-specific antibody prevents graft-versus-host disease in mice. J. Immunol. 164, 4564-4568 (2000).

36. Greenberg, P. D. \& Riddell, S. R. Deficient cellular immunity-finding and fixing the defects. Science 285, 546-551 (1999).

37. Krenger, W., Hill, G. R. \& Ferrara, J. L. Cytokine cascades in acute graft-versus-host disease. Transplantation 64, 553-558 (1997).

38. Shlomchik, W. D. et al. Prevention of graft versus host disease by inactivation of host antigenpresenting cells. Science 285, 412-415 (1999).

39. Riddell, S. R. et al. Restoration of viral immunity in immunodeficient humans by the adoptive transfer of T cell clones. Science 257, 238-241 (1992).

40. Walter, E. A. et al. Reconstitution of cellular immunity against cytomegalovirus in recipients of allogeneic bone marrow by transfer of T-cell clones from the donor. N. Engl. J. Med. 333, 1038-1044 (1995).

41. Hunt, D. F. et al. Characterization of peptides bound to the class I MHC molecule HLA-A2.1 by mass spectrometry. Science 255, 1261-1263 (1992).

42. Goulmy, E. Human minor histocompatibility antigens: new concepts for marrow transplantation and adoptive immunotherapy. Immunol. Rev. 157, 125-140 (1997).

43. den Haan, J. M. et al. The minor histocompatibility antigen HA-1: a diallelic gene with a single amino acid polymorphism. Science 279, 1054-1057 (1998).

44. Mutis, T. et al. Feasibility of immunotherapy of relapsed leukemia with ex vivo-generated cytotoxic T lymphocytes specific for hematopoietic system-restricted minor histocompatibility antigens. Blood 93, 2336-2341 (1999).

45. Warren, E. H., Greenberg, P. D. \& Riddell, S. R. Cytotoxic T-lymphocyte-defined human minor histocompatibility antigens with a restricted tissue distribution. Blood 91, 2197-2207 (1998).

46. Warren, E. H., Gavin, M., Greenberg, P. D. \& Riddell, S. R. Minor histocompatibility antigens as targets for T-cell therapy after bone marrow transplantation. Curr. Opin. Hematol. 5, 429-433 (1998)

47. Lapidot, T. et al. A cell initiating human acute myeloid leukaemia after transplantation into SCID mice. Nature 367, 645-648 (1994).

48. Bonnet, D., Warren, E. H., Greenberg, P. D., Dick, J. E. \& Riddell, S. R. CD8 ${ }^{+}$minor histocompatibility antigen-specific cytotoxic $\mathrm{T}$ lymphocyte clones eliminate human acute myeloid leukemia stem cells. Proc. Natl Acad. Sci. USA 96, 8639-8644 (1999).

49. Dermime, S. et al. Lack of T-cell-mediated recognition of the fusion region of the pml/RAR-alpha hybrid protein by lymphocytes of acute promyelocytic leukemia patients. Clin. Cancer Res. 2, 593-600 (1996).

50. Chen, W., Qin, H., Reese, V. A. \& Cheever, M. A. CTLs specific for bcr-abl joining region segment peptides fail to lyse leukemia cells expressing p210 bcr-abl protein. J. Immunother. 21, 257-268 (1998)

51. Sturrock, A., Franklin, K. F. \& Hoidal, J. R. Human proteinase-3 expression is regulated by PU.1 in conjunction with a cytidine-rich element. J. Biol. Chem. 271, 32392-32402 (1996).

52. Sturrock, A. B. et al. Structure, chromosomal assignment, and expression of the gene for proteinase-3 The Wegener's granulomatosis autoantigen. J. Biol. Chem. 267, 21193-21199 (1992).

53. Molldrem, J. J. et al. Cytotoxic T lymphocytes specific for a nonpolymorphic proteinase 3 peptide preferentially inhibit chronic myeloid leukemia colony-forming units. Blood 90, 2529-2534 (1997).

54. Molldrem, J. et al. Targeted T-cell therapy for human leukemia: cytotoxic T lymphocytes specific for a peptide derived from proteinase 3 preferentially lyse human myeloid leukemia cells. Blood $\mathbf{8 8}$, 2450-2457 (1996).

55. Molldrem, J. J. et al. Evidence that specific T lymphocytes may participate in the elimination of chronic myelogenous leukemia. Nature Med. 6, 1018-1023 (2000).

56. Inoue, K. et al. Aberrant overexpression of the Wilms tumor gene (WT1) in human leukemia. Blood 89, 1405-1412 (1997).

57. Bergmann, L. et al. High levels of Wilms' tumor gene (wt1) mRNA in acute myeloid leukemias are associated with a worse long-term outcome. Blood 90, 1217-1225 (1997).

58. Gao, L. et al. Selective elimination of leukemic CD34+ progenitor cells by cytotoxic T lymphocytes specific for WT1. Blood 95, 2198-2203 (2000).

59. Borrello, I. et al. Sustaining the graft-versus-tumor effect through posttransplant immunization with granulocyte-macrophage colony-stimulating factor (GM-CSF)-producing tumor vaccines. Blood 95 3011-3019 (2000).

60. Appelbaum, F. R. Antibody-targeted therapy for myeloid leukemia. Semin. Hematol. 36, 2-8 (1999).

\section{Acknowledgements}

The author thanks P. Greenberg, R. Storb, J. Hansen, E. Petersdorf, S. Riddell and C. Anasetti for the development of many of the concepts reviewed in this manuscript. 